\title{
Polymerization of Allyl Alcohol by Radiation to Obtain Microencapsulated Structure
}

\author{
Ali UsAnMAZ and Süreyya (Zengin) SARICILAR \\ Department of Chemistry, Middle East Technical University, \\ Ankara, Turkey
}

(Received August 22, 1988)

\begin{abstract}
Allyl alcohol was polymerized by radiation under various conditions. The limiting conversions were about $30 \%$ in bulk, $35 \%$ when containing 0.03 mole fraction $\mathrm{AlCl}_{3}$ and $50 \%$ when water was contained at $27 \%(\mathrm{v} / \mathrm{v})$. Irradiation was done with Co- 60 gamma rays at room temperature and under vacuum. The presence of oxygen did not cause any change in the reaction rate. Molecular weights were determined by viscosity and cryoscopic methods. $K$ and $\alpha$ values were found to be $3.57 \times 10^{-4}$ and 0.62 for solutions in methanol at $25^{\circ} \mathrm{C}$. The polymers up to about $10 \%$ conversion were viscous liquids having microcapsular structures: at high conversions, they became hard and glassy. The microencapsulated structures were also retained in solutions in methanol, acetone, and isopropyl alcohol. The samples were insoluble in water, benzene, and toluene.
\end{abstract}

KEY WORDS Allyl Alcohol/ Microcapsule / Microscopy / Polymerization

/ Chemical Catalyst / Intrinsic Viscosity / Degradative Transfer /

Allyl alcohol $\mathrm{CH}_{2}=\mathrm{CH}-\mathrm{CH}_{2} \mathrm{OH}$, usually polymerizes slowly and gives oligomers because of degradative transfer to the monomer. The thermal polymerization with peroxide is reported to give oligomers containing 5-10 monomeric units. ${ }^{1}$ Goodman et al. ${ }^{2}$ reported polymers with molecular weights of 2000 and showing some crystallinity when allyl alcohol was polymerized by $\mathrm{BF}_{3}$ at low temperature. Clay et al. $^{3}$ reported the formation of acrolein, hydroxyacetyl aldehyde, formaldehyde and hydroxyperoxide when allyl alcohol-water solutions were irradiated with gamma rays. However, Dolmatov and Polak ${ }^{4}$ reported the formation of a polymer with relatively high molecular weight when an aqueous solution of monomer was irradiated. The polymerization by radiation of monomer containing a salt of main groups elements IA and IIA metals or mineral acids gives a solid, amorphous polymer. ${ }^{5}$ According to Kargin et al..$^{5}$ the rate of radiation-induced polymerization was increas- ed from $0.15 \%$ to $1.5 \%$ per hours when $27 \%$ $\mathrm{CaCl}_{2}$ was added to monomer. Under these conditions, the molecular weight also increased from 1000 to $5 \times 10^{5}$. The reactivities of allyl monomers in radical polymerization were studied by Zubov et al. ${ }^{6}$ They showed that the protonation or complexing of the alcohol group in allyl alcohol changes degradative chain transfer into effective chain transfer. The rate of polymerization increases with increase of the proportion of mineral acids such as $\mathrm{H}_{3} \mathrm{PO}_{4}$.

The microcapsular structure of allyl alcohol has not been reported before. This type of structure may have many commercial and research applications. The $K$ and $\alpha$ values have not been reported for the polymer. In this work, the polymerization of allyl alcohol by radiation is studied in more detail and the effects of various additives are investigated. The conditions of polymerization giving microencapsulation are also studied. 


\section{EXPERIMENTAL}

Allyl alcohol (Aldrich) was purified by distillation at reduced pressure $\left(58^{\circ} \mathrm{C} / 10 \mathrm{~mm}\right.$ $\mathrm{Hg}$ ). It was used immediately after distillation. $N, N$-Dimethylformamide, (DMF), was of Merck reagent grade and kept over anhydrous $\mathrm{CuSO}_{4}$ overnight and refluxed; it was distilled at reduced pressure. Other organic solvents were purified by distillation.

Polymerization was initiated by Co- 60 gamma rays at a dose rate of $1 \times 10^{5} \mathrm{rad} \mathrm{h}^{-1}$ for the desired time. About $2 \mathrm{ml}$ of freshly distilled monomer were placed in a pyrex irradiation tube of $16 \mathrm{~mm}$ diameter. The sample tubes were evacuated at $10^{-4}-10^{-5}$ $\mathrm{mmHg}$ for about $5-6 \mathrm{~h}$, and then sealed and placed in the irradiation source for the desired time. The unpolymerized polymer was removed by freeze-drying and polymer samples were dried to constant weight. The conversions were calculated gravimetrically.

The viscosities of the polymer samples were measured in methanol at $25^{\circ} \mathrm{C}$. The number average molecular weights of some samples were determined cryoscopically using DMF as solvent. A polystyrene sample with a numberaverage molecular weight of 4800 was used as the standard.

The microcapsular structures were observed by optical microscopy and photography of some samples in a polarizing microscope.

\section{RESULTS AND DISCUSSION}

\section{Polymerization of Allyl Alcohol}

Allyl alcohol in bulk or in a mixture containing water or aluminum chloride was polymerized by radiation. The conversion $v s$. irradiation time for the bulk polymerization is shown in Figure 1a. Conversion increases almost linearly up to $10 \%$ and levels off at about $30 \%$. A conversion-time curve for polymerization of monomer containing 0.03 mole fraction $\mathrm{AlCl}_{3}$ is shown in Figure $1 \mathrm{~b}$. The curve is quite similar to that for monomer in bulk but

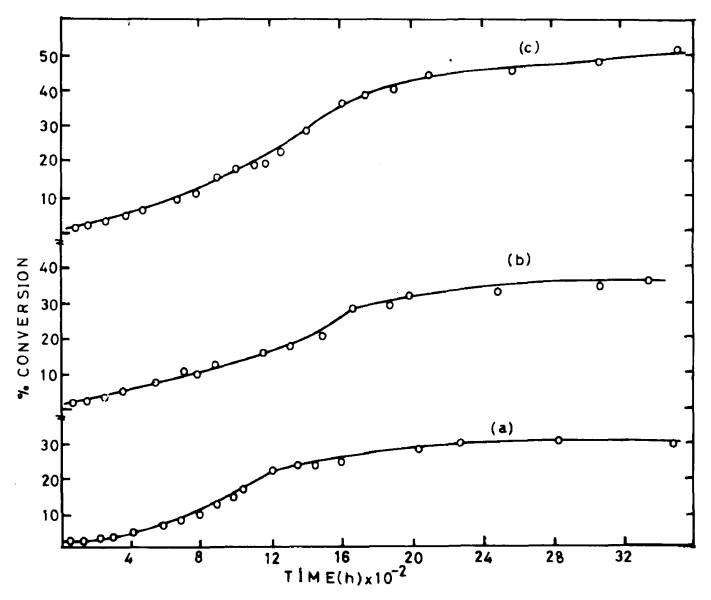

Figure 1. Polymerization of allyl alcohol by radiation at room temperature: (a), bulk monomer; (b), monomer containing $0.03 \mathrm{~mol}$ fraction of $\mathrm{AlCl}_{3}$; (c), monomer containing $27 \%(\mathrm{v} / \mathrm{v}) \mathrm{H}_{2} \mathrm{O}$.

the limiting conversion in this case is about $35 \%$. The relationship of conversion to mole fraction of $\mathrm{AlCl}_{3}$ was also studied. The mole fraction of $\mathrm{AlCl}_{3}$ was increased in steps up to 0.24 which corresponds to saturation. The conversions for all concentrations were the same. Therefore it was concluded that the presence of $\mathrm{AlCl}_{3}$ in the polymerization medium does not show a great effect except a small increase in limiting conversion. This is different from the effect observed by Kargin et al. for $\mathrm{CaCl}_{2}$ and other salts of metals of groups IA and IIA. The results show that $\mathrm{AlCl}_{3}$ does not make a similar complex with allyl alcohol that might change degradative chain transfer into chain transfer, as reported. ${ }^{5,6}$

A plot of conversion against time of irradiation for allyl alcohol containing $27 \%(\mathrm{v} / \mathrm{v})$ water is shown in Figure 1c. The conversion increases to about $10 \%$ for the bulk system, but the limiting conversion increases to about $50 \%$. This effect may be due to solvation of the alcohol group and decrease in the rate of degradative chain transfer, similar to protonation by mineral acids. ${ }^{6} \mathrm{~A}$ second and most probable effect may be due to dilution which 
has not been considered by other workers. ${ }^{4-6}$ The polymer is soluble in monomer and no phase separation can be observed. Therefore its molecules which have closely spaced- $\mathrm{CH}_{2} \mathrm{OH}$ side groups form a microcapsule structure because of high hydrogen bonding. The dispersed microcapsules decrease the mobility of the monomer molecules. After about $10 \%$ conversion, the increase in molecular weight and number of microcapsules causes intermolecular hydrogen bonding to be more effective, resulting in glassy state formation. This type of structure decreases the mobility of molecules further and limiting conversion is reached. The presence of water in the polymerization medium makes the monomer molecules more mobile due to the dilution. This is also substantiated by increase in limiting conversion by increase of water content up to azeotropic point where it gives maximum value. This has also been reported by Dolmatov and Polak. ${ }^{4}$

The polymerization of allyl alcohol was repeated for samples open to the air up to about $15 \%$ conversion. The results were identical to those under vacuum conditions. Therefore, the presence of oxygen in the reaction medium does not have any noticeable effect on the polymerization at these conversions.

The polymers obtained in all cases were soluble in alcohols, acetone, and DMF but insoluble in water, benzene, and toluene.

The ESR investigation did not give a recognizable chain propagating radical. From this along with the low activated energy of 12.5 $\mathrm{kJ} \mathrm{mol}^{-1}$ reported ${ }^{4}$ and the absence of retardation effect of oxygen, the mechanism of polymerization probably is ionic rather than radicalic. However, more work has to be done to find the exact mechanism of polymerization.

The structure of polymers obtained under various conditions were studied by IR spectra. In all cases, only - $\mathrm{OH}$ peaks were observed in such a high relative intensity so that the peaks due to $-\mathrm{CH}_{2}-$ and other groups could not be observed. The polymerization is most probably carried out by opening of carbon- carbon double bonds. However, the possible isomerization of molecules cannot completely be sustained.

\section{Molecular Weight Determination}

The molecular weights of poly(allyl alcohol) obtained by radiation under various conditions were determined by the viscosity method. The number-average molecular weights for several samples were determined cryoscopically. The $\log [\eta] v s . \log \bar{M}_{n}$ plot is given in Figure 2. From the intercept and slope of the straight line, $K$ and $\alpha$ were found as $3.57 \times$ $10^{-4}$ and 0.62 , respectively. Therefore, the relation between the intrinsic viscosity and number average molecular weight is given as follows for solutions in methanol at $25^{\circ} \mathrm{C}$.

$$
[\eta]=3.57 \times 10^{-4} \bar{M}_{n}^{0.62}
$$

The molecular weight were calculated from measured intrinsic viscosities using this relation, and the results are plotted $v s$. conversion in Figure 3. The molecular weight in all cases increases, reaches a maximum, then decreases and levels off at a value of $3000-6000$. The position of the maximum depends upon the conditions during polymerization. The maximum is at about $20 \%$ conversion for polymers obtained from monomer containing $\mathrm{H}_{2} \mathrm{O}$, and at about $10 \%$ conversion for bulk and monomer containing $\mathrm{AlCl}_{3}$. The highest molecular weight of about 33000 was obtained from bulk polymerization; for the monomer containing

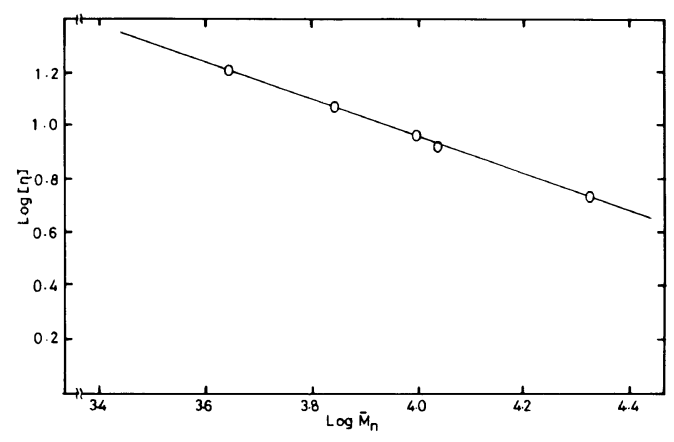

Figure 2. Intrinsic viscosity-number average molecular relation for poly(allyl alcohol). 


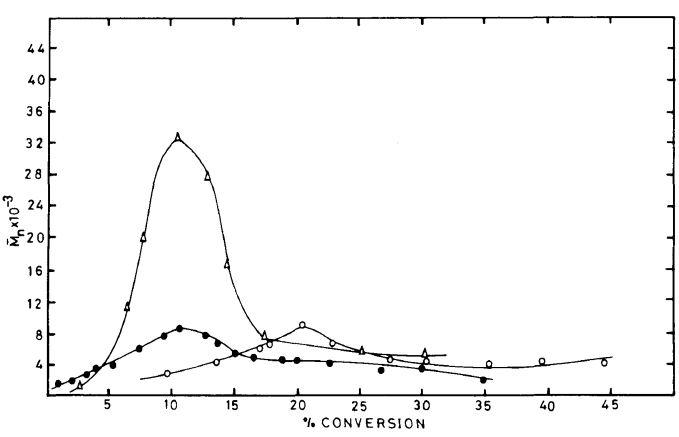

Figure 3. Molecular weight change with respect to percent conversion for poly(allyl alcohol): $\triangle$, bulk; $\bigcirc$, containing $\mathrm{H}_{2} \mathrm{O}$; $\boldsymbol{O}$, containing $\mathrm{AlCl}_{3}$.

$\mathrm{H}_{2} \mathrm{O}$, the maximum is about 10000 and for monomer containing $\mathrm{AlCl}_{3}$ it is about 8000 . The shift in maxima for molecular weightconversion relation of polymerization of samples containing $\mathrm{H}_{2} \mathrm{O}$ also substantiated the dilution effect of water.

\section{Microencapsulation of Poly(allyl alcohol)}

The polymer samples obtained under all conditions up to about $10 \%$ conversion have microcapsular structures when studied with the polarizing microscope either in bulk form or in solution in alcohol and acetone. The alcohols used were methanol and isopropanol. The microcapsular structures were retained after the solvent was removed by evaporation. The size of a microcapsule varies very little with conversion but the largest was found for conversions close to $10 \%$. At higher conversions, the samples were hard and glassy and microencapsulation could not be observed. After drying, the microcapsules retained their shape and were heat resistant up to about $130^{\circ} \mathrm{C}$. Photographs taken with a polarizing microscope are shown in Figure 4 for samples in methanol solution, in Figure 5 for samples in acetone solution and in Figure 6 for bulk samples of viscous oily polymer. In all cases, the magnification is 100 . The microcapsules deform at temperatures over $130^{\circ} \mathrm{C}$. The polymer samples dehydrate at temperatures over $230^{\circ} \mathrm{C}$ and are then heat resistant up to about

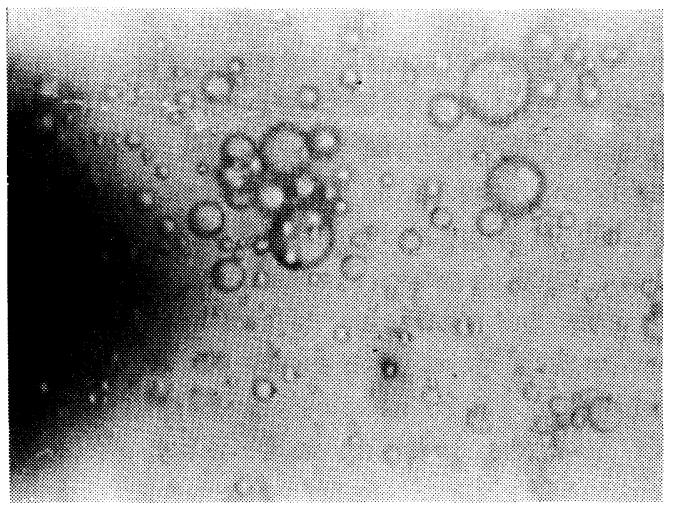

Figure 4. Microencapsulated poly(allyl alcohol) dispersed in methanol solution $(\times 100)$.

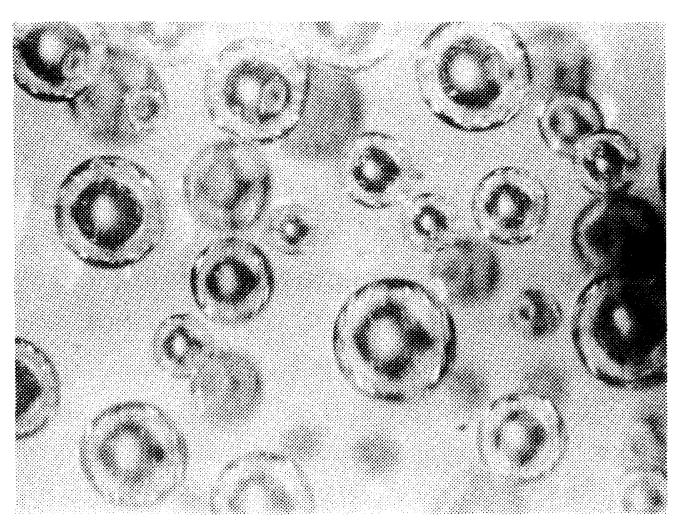

Figure 5. Microencapsulated poly(allyl alcohol) dispersed in acetone solution $(\times 100)$.

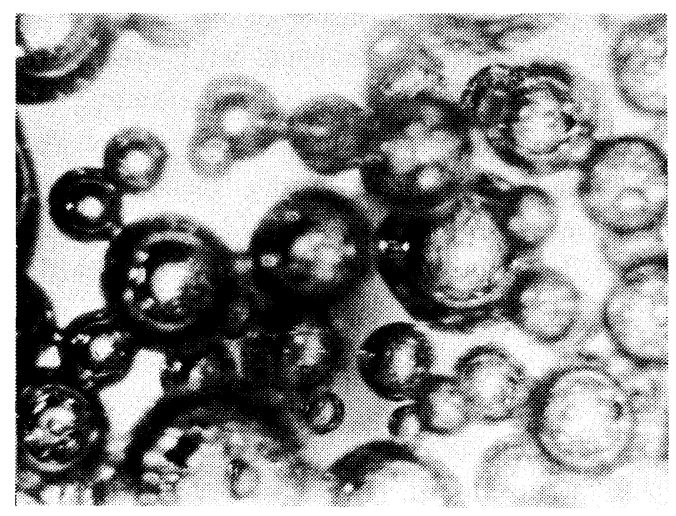

Figure 6. Microencapsulated poly(allyl alcohol) in bulk form $(\times 100)$.

$350^{\circ} \mathrm{C}$ which decompose. The phhtographs of samples kept at $150^{\circ} \mathrm{C}$ and $200^{\circ} \mathrm{C}$ for about three hours are shown in Figures 7 and 8 (at 


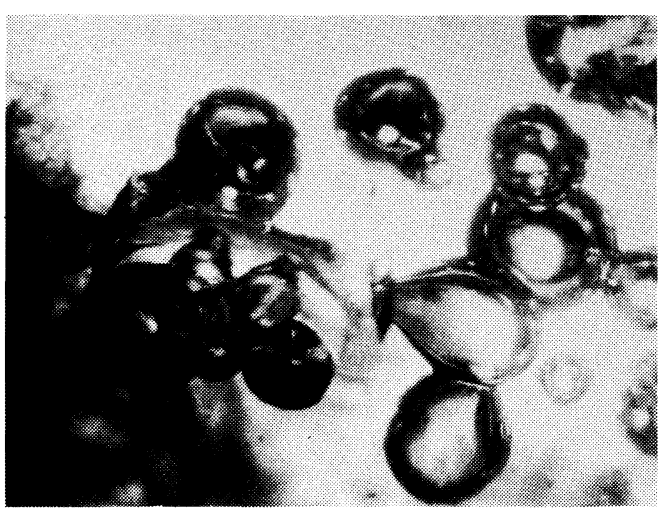

Figure 7. Microencapsulated poly(allyl alcohol) deformation at $150^{\circ} \mathrm{C}(\times 300)$.

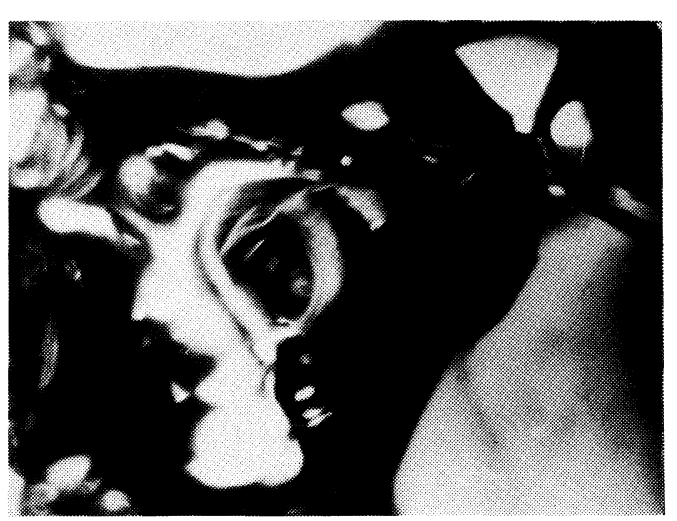

Figure 8. Microencapsulated poly(allyl alcohol) complete deformation at $200^{\circ} \mathrm{C}(\times 300)$.

300 magnification) respectively. Figure 7 shows severe deformation of microcapsules at $150^{\circ} \mathrm{C}$. The deformation is complete in Figure 8 without any dehydration of the sample.

Microencapsulation is possible for low molecular weight (about 300-6000) polymers but glassy polymers are more favored for high molecular weights. The polymer molecules are expected to be solvated by monomer and water molecules, but in fact the polymer is insoluble in water. This should be related to the conformation of polymer molecules which make them behave like micelle forming molecules. In order to understand the mechanism of encapsulation better, the conformation and configuration of polymers have to be studied well.

\section{CONCLUSIONS}

In this work, it has been shown that allyl alcohol can be polymerized by radiation to give low molecular weight polymers with microencapsular structure. The microencapsulated samples can be dispersed in certain solvents such as methanol and acetone. This may be important polymer application.

Polymerization to a limited conversion required about a 20 Mrad total dose, and requires very prolonged irradiation when the dose rate is low. The time of irradiation can be reduced by high dose rates. More work will be done to investigate the effects of dose rate on both the rate of polymerization and microencapsulation. The additives in this work did not increase the molecular weights of the polymers or rate of polymerization. This can be due to certain effective concentrations of additives and also not all types of metal halides can make complexes with monomer to increase the reactivity toward polymerization.

Acknowledgement. This work was partly supported by TUBITAK (Turkish National and Scientific Research Council) through a TUMKA unit.

\section{REFERENCES}

1. H. Dannenberg and D. E. Adelson, Br. Patent 566344; U.S. Patent 2541155 (Shell).

2. I. Goodman and J. Mather, Br. Patent 854207 (ICI), Nov. 16, 1960.

3. P. G. Clay, J. Weiss, and J. Whiston, Proc. Chem. Soc., 125 (1959).

4. S. A. Dolmatov and L. S. Polak, Neftekhimiya, 3, 689 (1963) (Chem. Abstr., 60, 3102e); Kinetika $i$ Kataliz, 6, 797 (1965) (Chem. Abstr., 64, 3691).

5. V. A. Kargin, L. S. Polak, V. A. Kabanov, X. P. Zubov, and V. F. Pankova, U.S. Patent 3666740, Appl. Aug. 14, 1969.

6. V. P. Zubov, M. V. Kumar, M. N. Masterova, and V. A. Kabanow, J. Macromol. Sci.-Chem., A13, 111 (1979). 